\title{
Focal adhesion kinase is involved in the migration of human osteosarcoma cells
}

\author{
SITAN FENG ${ }^{1 *}, \mathrm{XIN} \mathrm{SHI}^{2 *}, \mathrm{KE} \mathrm{REN}^{3 *}$, SUJIA WU $^{2}$ and XIAOLIANG SUN ${ }^{3}$ \\ ${ }^{1}$ Department of Orthopedics, Ruikang Hospital Affiliated to Guangxi University of Chinese Medicine, Nanning, \\ Guangxi 530011; ${ }^{2}$ Department of Orthopedics, Jinling Hospital, School of Medicine, Nanjing University, Nanjing, \\ Jiangsu 210002; ${ }^{3}$ Department of Orthopedics, The Third Affiliated Hospital of Soochow University, \\ The First People's Hospital of Changzhou, Changzhou, Jiangsu 213003, P.R. China
}

Received May 7, 2014; Accepted January 19, 2015

DOI: $10.3892 / \mathrm{ol} .2015 .3131$

\begin{abstract}
The aim of the present study was to analyze the expression of focal adhesion kinase (FAK) in osteosarcoma (OS) cell lines with different migration abilities in order to determine the role of FAK in migration. A number of different 143B subclone cell lines (A1, A2, A3, A4 and A5) were obtained by a limiting dilution method, and the expression of FAK was detected using western blot analysis. The role of FAK in the migration of OS cells was investigated using small interfering RNA (siRNA), and the ratio of the number of lamellipodia was compared by immunofluorescence staining. The A2 and A3 OS 143B subclone cell lines demonstrated a stronger migration ability and exhibited higher FAK expression compared with the A1 cell line $(\mathrm{P}<0.05)$. Following transfection with FAK-siRNA, the migration ability of the A3 cells was significantly decreased $(\mathrm{P}<0.05)$, and the ratio of the number of lamellipodia formed was reduced from 35 to $11 \%(\mathrm{P}<0.05)$. In conclusion, the level of FAK expression was higher in the cell lines with a stronger migration ability. FAK affects the migration ability of OS cells by suppressing the formation of lamellipodia.
\end{abstract}

\section{Introduction}

Osteosarcoma (OS) is the most frequently diagnosed primary malignant bone tumor, accounting for $2.4 \%$ of all childhood malignancies. The average age of onset for OS is

Correspondence to: Dr Ke Ren, Department of Orthopedics, The Third Affiliated Hospital of Soochow University, The First People's Hospital of Changzhou, 185 Juqian, Changzhou, Jiangsu 213003, P.R. China

E-mail: renke1977@sohu.com

${ }^{*}$ Contributed equally

Key words: osteosarcoma, subclone cell lines, focal adhesion kinase, migration, lamellipodia
10-20 years old $(1,2)$. As the primary lesion can be surgically removed, distant migration is the major cause of OS-associated mortality. However, the mechanisms of migration remain to be elucidated. Therefore, the study of OS migration is important for diagnostic and treatment strategies.

Focal adhesion kinase (FAK) is a non-receptor tyrosine kinase with tyrosine kinase activity (3). The autophosphorylation of FAK at tyrosine 397 can activate downstream target proteins, mediate a number of signal pathways and has a central role in a number of cellular events. In addition, FAK is involved in tumor cell proliferation, survival, apoptosis, spreading, migration and invasion. A number of previous studies have revealed that FAK is overexpressed in a variety of malignant tumors, including breast, lung, prostatic and colorectal cancer, hepatocellular carcinoma and pancreatic adenocarcinoma (4). The overexpression of FAK has an important role in tumor metastasis and confers a poor patient prognosis. However, the role of FAK in the migration of OS remains unclear.

In the present study, a limiting dilution method was used to analyze the expression of FAK in different OS cell lines. In addition, small interfering RNA (siRNA) was used to examine the role of FAK in OS cell migration.

\section{Materials and methods}

Materials. The OS 143B cell lines were obtained from the American Type Culture Collection (Manassas, VA, USA). The RPMI 1640 medium was purchased from the Beijing Wensent Co. (Beijing, China), the characterized fetal bovine serum (FBS) was obtained from TransGen Biotech Inc., (Beijing, China) and trypsin was purchased from Amresco LLC (Solon, OH, USA). The FAK polyclonal rabbit anti-human and mouse anti-human $\alpha$-tubulin primary antibodies were purchased from Sigma-Aldrich (St. Louis, MO, USA), and the anti-mouse immunoglobulin $\mathrm{G}$ ( $\mathrm{IgG}$ ) and anti-rabbit IgG secondary antibodies were purchased from GE Healthcare Life Sciences (Logan, UT, USA). The FAK siRNA sequence (GGUUCAAGCUGGAUUAUUTT) and the negative control siRNA sequence (UUCUCCGAACGUGUCACGUTT) were obtained from the Shanghai GenePharma Co., Ltd. (Shanghai, China). The phalloidin, DAPI antibodies, transfection reagents, Lipofectamine 2000, Opti medium and staining secondary 
antibodies were purchased from Invitrogen Life Technologies (Carlsbad, CA, USA).

Cell culture. The OS 143B cell lines were cultured in RPMI 1640 medium with 10\% FBS, $50 \mathrm{U} / \mathrm{ml}$ penicillin and $50 \mu \mathrm{g} / \mathrm{ml}$ gentamicin at $37^{\circ} \mathrm{C}$ in a $5 \% \mathrm{CO}_{2}$ incubator in a humidified atmosphere. The medium was changed every three days. Upon reaching $80 \%$ confluence, the cells were digested with $0.25 \%$ trypsin and passaged. All cells were in the logarithmic growth phase.

Monoclonal separation of the OS $143 B$ cell lines. The OS 143B cell lines were diluted into 96 -well plates at 1 cell/well using the limiting dilution method. The monoclonal cells in the wells were observed and recorded using an inverted microscope (Eclipse Ti-U; Nikon Corporation; Tokyo, Japan). Next, the cells were cultured in conditioned medium with $20 \%$ FBS and $50 \mathrm{U} / \mathrm{ml}$ penicillin and $50 \mu \mathrm{g} / \mathrm{ml}$ streptomycin, and kept at $37^{\circ} \mathrm{C}$ in a $5 \% \mathrm{CO}_{2}$ incubator in a humidified atmosphere for 2-3 weeks. Upon covering a third of the well, the cells were transferred to 24-well plates to expand in culture, then to 6 -wells plates and finally to a dish.

OS cell migration detection using the wound healing assay. In total, five small dots were drawn with a marker pen in parallel to scratches made on the back of a 12-well plate. An equal number of cells from each $143 \mathrm{~B}$ subclone cell line were then inoculated and cultured at $37^{\circ} \mathrm{C}$ in a $5 \% \mathrm{CO}_{2}$ incubator in a humidified atmosphere. When the cell monolayers had reached 90-100\% confluency, single scratches were made using $200-\mu 1$ tips along the bottom of the culture plates. The cellular debris were rinsed three times with phosphate-buffered saline (PBS), added to the medium and then analyzed with an inverted microscope (Eclipse Ti-U; Nikon Corporation). The 'A' value was taken as the distance of the scratch area in the vicinity of each dot, and the ' $\mathrm{B}$ ' value was taken after the cells had been cultured for $24 \mathrm{~h}$ or subsequent to scratch healing. Relative migration was calculated according to the following equation: Relative migration $=(\mathrm{A}-\mathrm{B}) / \mathrm{A}$. In the same period, the relative displacement of the subclone cells indicated the migration ability. The experiment was performed in triplicate.

Western blot analysis. An equal number of cells with different migration abilities from each OS subclone cell line were plated into 6 -well plates at $37^{\circ} \mathrm{C}$ in a $5 \% \mathrm{CO}_{2}$ incubator with a humidified atmosphere. When the cells had reached $80 \%$ confluency, the medium was removed. Next, the cells were washed twice with PBS and added to SDS. The cells were then scraped and boiled for $5 \mathrm{~min}$. Following centrifugation at $10,000 \mathrm{x} \mathrm{g}$ for $5 \mathrm{~min}$, the supernatant was stored for subsequent use. Equal quantities of the samples were separated by SDS-PAGE and then transferred onto polyvinylidene difluoride membranes. Subsequent to blocking with 5\% skimmed milk, the membranes were incubated overnight at $4{ }^{\circ} \mathrm{C}$ with primary antibodies against FAK (dilution, 1:400) and $\alpha$-tubulin (dilution, 1:800). Next, the membranes were washed three times with Tris-buffered saline and Tween 20 (TBST) and incubated with the secondary antibodies for $2 \mathrm{~h}$ at room temperature. The membranes were then washed a further three times with TBST, for $10 \mathrm{~min}$ each time. Subsequent to development and fixing, the blots were exposed to X-ray film in order to observe the protein expression level.

Subclone cell lines with strong migration ability transfected with FAK-siRNA. The subclone cell lines with strong migration abilities were separated and total of $2 \times 10^{5}$ cells were plated into 6-well plates at $37^{\circ} \mathrm{C}$ in a humidified atmosphere of $5 \%$ $\mathrm{CO}_{2}$ for $24 \mathrm{~h}$. The cells grew to $40-60 \%$ confluency within the $24 \mathrm{~h}$. The control cells were transfected with siRNA, and the experimental cells with siRNA specific to FAK at concentrations of 30,50 and $60 \mathrm{nM} / 1$. The control cells were transfected with Lipofectamine 2000 according to the manufacturer's instructions. The FAK-siRNA and control siRNA were mixed with Lipofectamine 2000 and left to sit for $20 \mathrm{~min}$ prior to being added to 6 -well plates at $37^{\circ} \mathrm{C}$ in $5 \% \mathrm{CO}_{2}$ incubator in a humidified atmosphere. The culture medium was changed after $6 \mathrm{~h}$. The transfection level, migration ability and pseudopodia formation were examined after $48 \mathrm{~h}$.

Lamellipodia were counted using an immunofluorescence staining method. The siRNA and FAK-siRNA at a concentration of $50 \mathrm{nM} / 1$ were added to the cell lines with strong migration abilities for $48 \mathrm{~h}$. Next, the cells were fixed with $2 \%$ paraformaldehyde at room temperature for $8 \mathrm{~h}$ and then washed with PBS three times for 5 min each. Staining with fluorescein isothiocyanate (FITC)-phalloidin (dilution, 1:500) and DAPI (dilution, 1:500) was then performed at room temperature for $60 \mathrm{~min}$. The cells were then washed three times with PBS for 5 min each. Next, anti-fluorescence quenching liquid was added and the cells were examined under a fluorescence microscope (Delta Vision microscope, Applied Precision Inc., Issaquah, WA, USA). A total of 200 cells were used to calculated the number of lamellipodia and proportion of the total number. The experiment was performed in triplicate.

Statistical analysis. All data were analyzed using SPSS software version 13.0 (SPSS, Inc., Chicago, IL, USA). The results are presented as the mean \pm standard deviation. The data was analyzed by a one-way analysis of variance and $\chi^{2}$ test. $\mathrm{P}<0.05$ was considered to indicate a statistically significant difference.

\section{Results}

Separation of the OS $143 B$ subclone cell lines. Single cells were successfully isolated from $\sim 20$ in a 96-well plate. In total, 14 clones continued to grow successfully following transmission to the dish. The rest had undergone apoptosis or were unable to be passaged. Under the same culture conditions, two subclone cell lines underwent spontaneous cell death at passages 8-12, and so could not continue through passaging.

Separation of the OS $143 B$ subclone cell lines according to different migration abilities. In total, five subclone cell lines (A1, A2, A3, A4 and A5) under the same generation and with similar growth rates were selected for the scratch test. The results indicated that the migration abilities of A2 $(0.931 \pm 0.010)$ and A3 $(0.941 \pm 0.014)$ were significantly higher than A1 (0.458 \pm 0.019$), \mathrm{A} 4(0.618 \pm 0.015)$ and the other cell 
A

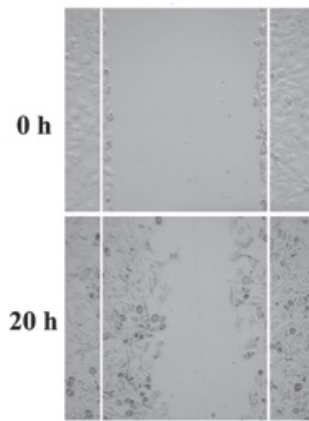

A1

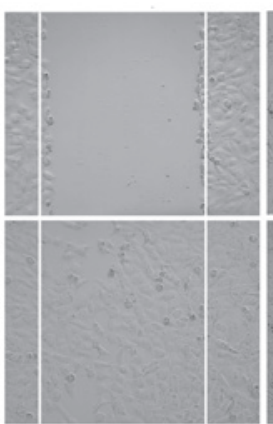

A2

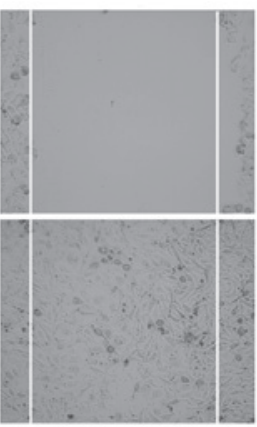

A3

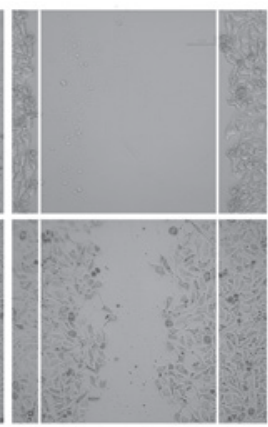

A4

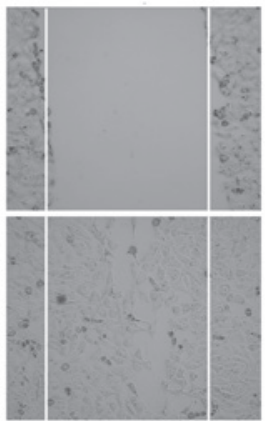

A5

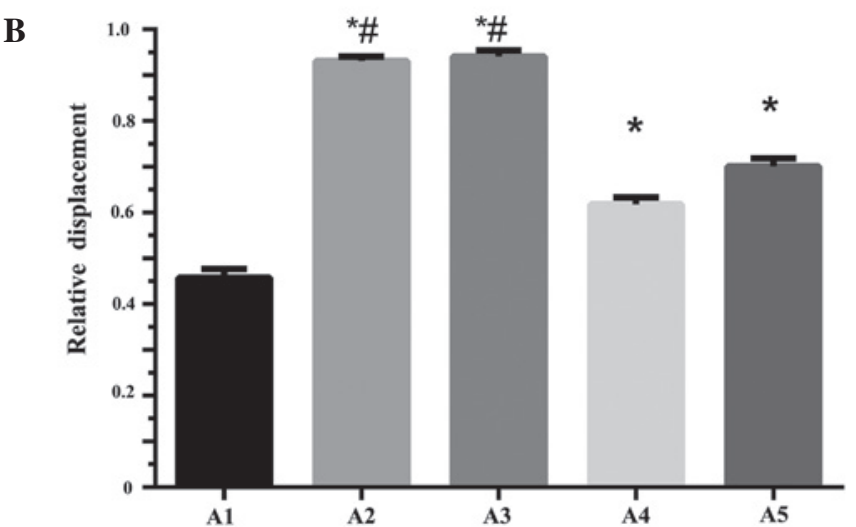

Figure 1. Separation of the osteosarcoma (OS) 143B cell lines with different migration abilities. (A) A wound healing assay was performed on the OS 143B subclone cell lines, A1, A2, A3, A4 and A5. (B) The relative displacement of the osteosarcoma 143B subclone cell lines, A1, A2, A3, A4 and A5. * P<0.05 vs. A1. ${ }^{\text {P }}>0.05, \mathrm{~A} 3$ vs. A2.

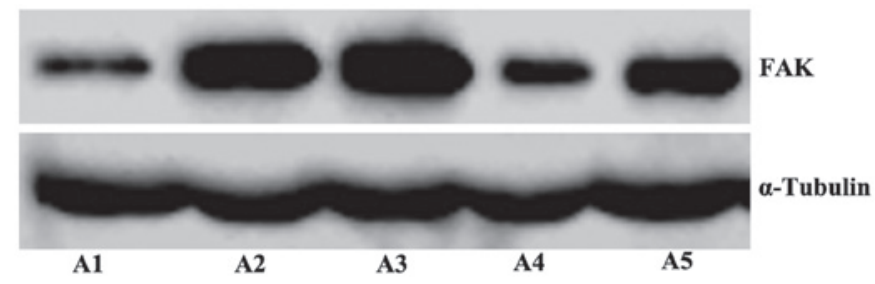

Figure 2. Western blot analysis revealing the expression of focal adhesion kinase (FAK) in the osteosarcoma 143B subclone cell lines, A1, A2, A3, A4 and A5, with different migration abilities.

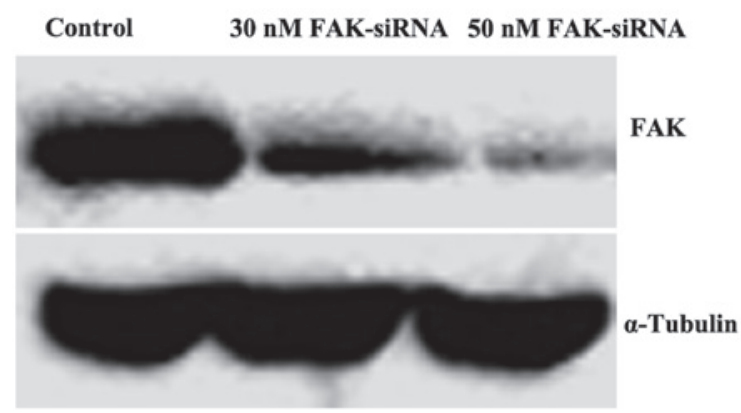

Figure 3. Western blot analysis revealing the low expression of focal adhesion kinase (FAK) in A3 osteosarcoma cells transfected with FAK-small interfering RNA (siRNA).

lines $(\mathrm{P}<0.05)$. Of the cell lines analyzed, A1 exhibited the slowest migration. No significant difference in migration was observed between A2 and A3 (P>0.05) (Fig. 1).
Expression of FAK in the OS $143 B$ subclone cell lines with different migration abilities. The results of the western blot analysis revealed that the expression of FAK in the A2 and A3 cell lines was markedly higher than that in the A1 cell line (Fig. 2).

Differential expression of FAK in OS cells transfected with FAK-siRNA. The results of the western blot analysis revealed that the expression of FAK in the experimental group was lower than that in the control group. The OS cells transfected with $50 \mathrm{nM} / 1$ FAK-siRNA scarcely expressed FAK, which indicated that the experiment had succeeded in knocking down the protein. FAK-siRNA, at a concentration of $>50 \mathrm{nM} / 1$, induced significant cytotoxicity. Therefore, FAK-siRNA at a concentration of $50 \mathrm{nM} / 1$ was selected for use in the experiments (Fig. 3).

Effect of FAK-knockdown on OS cell migration. The wound scratch assay demonstrated that following transfection with FAK-siRNA, the migration ability of the A3 cell line was significantly lower than that of the negative control group. After $24 \mathrm{~h}$, the relative displacement of the negative control cells was $0.942 \pm 0.025$, whereas the relative displacement of the cells transfected with FAK-siRNA was $0.322 \pm 0.01(\mathrm{P}<0.05)$ (Fig. 4).

Effect of FAK-knockdown on the lamellipodia formation of the OS cells. In contrast to the negative control group, silencing of FAK in the FAK-siRNA group lead to a sparse appearance of filaments surrounding the cells and a low fluorescence 


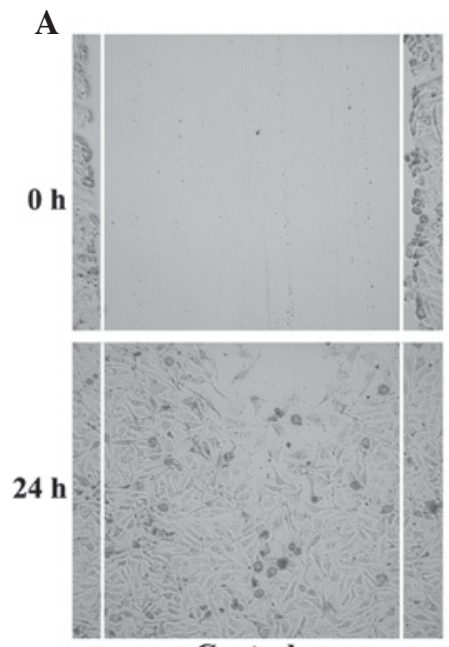

B

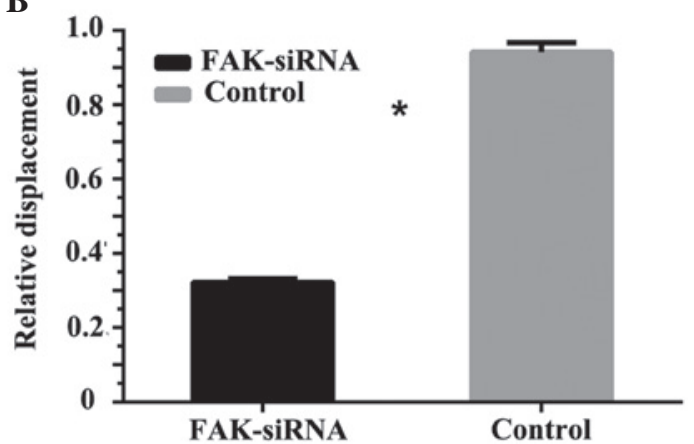

Figure 4. Effect of focal adhesion kinase (FAK) silencing on the migration of osteosarcoma (OS) cells. (A) A wound healing assay was performed using the control OS A3 cell line, and the OS A3 cell line transfected with FAK-small interfering RNA (siRNA). (B) The relative displacement of the control OS A3 cell line and the OS A3 cell line transfected with FAK-siRNA. "P $<0.05$ vs. control group.
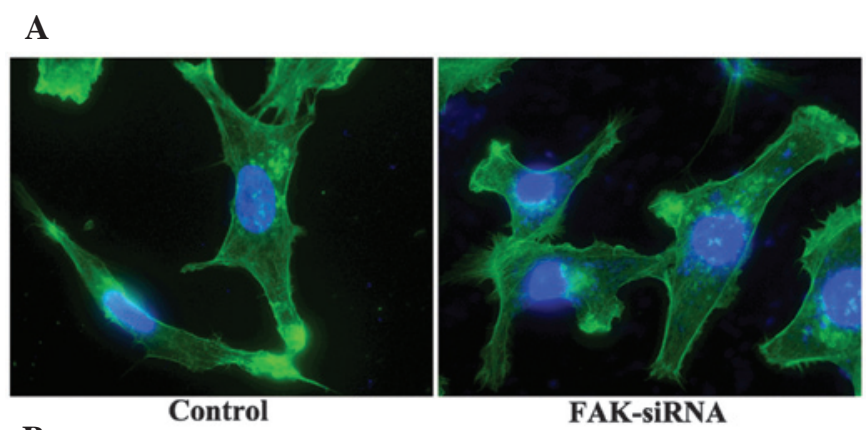

B

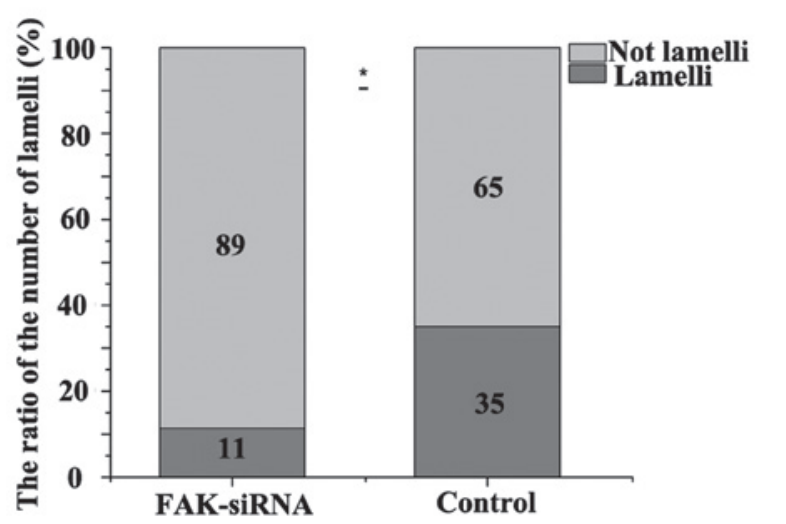

Figure 5. Effect of focal adhesion kinase (FAK) silencing on the formation of the lamellipodia. (A) Immunofluorescence staining was performed using the control and FAK-small interfering RNA (siRNA) groups. (B) The ratio of the number of lamellipodia in the control and FAK-siRNA groups. ${ }^{*} \mathrm{P}<0.05$ vs. control group. intensity. The ratio of the number of filopodia dropped from 33.3 to $12.8 \%$ in the FAK-siRNA group $(\mathrm{P}<0.05)$ (Fig. 5).

\section{Discussion}

OS is a disease with rapid progression, a poor prognosis, and a high morbidity and mortality rate $(5,6)$. Despite significant improvements in imaging techniques and the use of neoadjuvant chemotherapies, the five-year survival rate for OS patients remains at $65 \%(7,8)$. Furthermore, there have been no significant improvements in the rate of mortality compared with several years ago. Lung metastases are the main cause of mortality in patients with OS. Therefore, studying the metastatic mechanisms allows for effective assessment of the clinical prognosis and treatment of OS. In 1977, Fidler and Kripke (9) put forward the concept of heterogeneity as the basis of tumor migration. From then on, it was realized that primary malignant tumor cells exhibit heterogeneity (10). Within a tumor, the biological characteristics and metastatic behaviors of various subgroups are not identical. Furthermore, the invasion and migration of the subsets are inconsistent during the progression of the tumors. However, not all tumor cells have the ability of invasion and migration, and only certain subgroups have the potential to establish a stable, subline tumor metastatic phenotype. The present study analyzed OS cell lines (A1, A2, A3, A4 and A5) with different migration abilities using the limiting dilution method.

Tumor metastasis is a complex process. The three-step, molecular-level hypothesis for malignant cells was proposed by Liotta (11) and includes the following stages: i) Adhesion, ii) degradation and iii) migration and invasion. The migration of malignant cells largely determines their ability to transfer to the others. FAK is a widely-expressed, non-receptor protein tyrosine kinase located in the cytoplasm. Phosphorylation of FAK mediates signaling events, is widely involved in various biological processes within cells, and contributes to tumor progression, including invasion, metastasis and angiogenesis (8-11). FAK is closely associated with the migration of malignant cells. In the present study, the expression of FAK in the OS subclone A2 and A3 cell lines with high migration abilities was significantly higher than that in the A1 cell line with a low migration ability. Similarly, Chen et al (12) identified that the expression of FAK in hepatoma cells with a stronger migration and invasion ability was significantly higher than that in the hepatoma cells with weaker migration and invasion abilities. In the same study, the knockdown of FAK expression by siRNA affected the cellular migration of the hepatoma cells, a result also observed in human neuroblastoma (13) and melanoma (14) cells. The results of the present study also indicated that in the early migration process, reduced expression of FAK significantly decreased the number of lamellipodia. This result is consistent with the results of a study by Kwiatkowska et al (15), which found that the decreased expression of phosphorylated FAK (phospho-FAK) suppressed the migration of malignant glioma cells. The study also revealed that lamellipodia formation was significantly prolonged in the cells with low levels of phospho-FAK. These findings, in addition to those of the present study, suggest that the silencing of FAK affects the formation of lamellipodia. 
In summary, the results of the present study suggested that FAK has an important role in the migration of OS cells. FAK decreased the formation of lamellipodia, which in turn affected the migration ability of the cells. These findings provide a breakthrough in the study of the migration mechanisms involved in OS, and present a novel route for the clinical assessment, prognosis and treatment of the disease.

\section{Acknowledgements}

The study was supported by the Jiangsu Province Natural Science Foundation from the Science and Technology Department of Jiangsu Province of China (grant no. BK2012775), the Key Scientific and Technological Project of ChangzhouHealth Bureau (grant no. ZD201404), the Jiangsu Province Science and Technology Support Program of Social Development Projects (grant no. BE2013712).

\section{References}

1. Whelan JS: Osteosarcoma. Eur J Cancer 33: 1611-1619, 1997.

2. Murphey MD, Robbin MR, McRae GA, et al: The many faces of osteosarcoma. Radiographics 17: 1205-1231, 1997.

3. Lai IR, Chu PY, Lin HS, Liou JY, Jan YJ, Lee JC, Shen TL. Phosphorylation of focal adhesion kinase at Tyr397 in gastric carcinomas and its clinical significance. Am J Pathol 177: $1629-1637,2010$.

4. Golubovskaya VM: Focal adhesion kinase as a cancer therapy targy. Anticancer Agents Med Chem 10: 735-741, 2010.
5. Link MP, Goorin AM, Miser AW, et al: The effect of adjuvant chemotherapy on relapse-free survival in patients with osteosarcoma of the extremity. N Engl J Med 314: 1600-1606, 1986.

6. Wunder JS, Nielsen TO, Maki RG, et al: Opportunities for improving the therapeutic ratio for patients with sarcoma. Lancet Oncol 8: 513-524, 2007.

7. Messerschmitt PJ, Garcia RM, Abdul-Karim FW, et al Osteosarcoma. J Am Acad Orthop Surg 17: 515-27, 2009.

8. Parkes SE, Parke S, Mangham DC, et al. Fifty years of paediatric malignant bone tumours in the West Midlands, UK, 1957-2006: incidence, treatment and outcome. Paediatr Perinat Epidemiol 24: 470-478, 2010.

9. Fidler IJ and Kripke ML: Metastasis results from preexisting variant cells within a malignant tumor. Science 197: 893-895, 1977.

10. Mark M,Reinhat-King CA,Erickson D.Microfabricated physical spatial gradients for investigating cell migration and invasion dynamic. PLoS One 6: e20825, 2011.

11. Liotta LA: Cancer cell invasion and metastasis. Sci Am 266: 54-59, 62-63, 1992.

12. Chen JS, Huang XH, Wang Q, et al: FAK is involved in invasion and metastasis of hepatocellular carcinoma. Clin Exp Metastasis 27: 71-82, 2010.

13. Jones ML, Shawe-Taylor AJ, Williams CM and Poole AW: Characterization of a novel focal adhesion kinase inhibitor in human platelets. Biochem Biophys Res Commun 389: 198-203, 2009.

14. Tavora B, Batista S, Reynolds LE, et al: Endothelial FAK is required for tumour angiogenesis. EMBO Mol Med 2: 516-528, 2010.

15. Kwiatkowska A, Kijewska M, Lipko M, et al: Downregulation of Akt and FAK phosphorylation reduces invasion of glioblastoma cells by impairment of MT1-MMP shuttling to lamellipodia and downregulates MMPs expression. Biochim Biophys Acta 1813: 655-667, 2011. 